\title{
Transcranial magnetic theta-burst stimulation of the human cerebellum distinguishes absolute, duration-based from relative, beat-based perception of subsecond time intervals
}

\author{
Manon Grube ${ }^{1 *}$, Kwang-Hyuk Lee ${ }^{2}$,Timothy D. Griffiths' ${ }^{1}$, Anthony T. Barker ${ }^{3}$ and Peter W. Woodruff \\ Newcastle Auditory Group, Institute of Neuroscience, Medical School, Newcastle University, Framlington Place, Newcastle upon Tyne, UK \\ 2 Sheffield Cognition and Neuroimaging Laboratory, Academic Clinical Psychiatry, Department of Neuroscience, University of Sheffield, Sheffield, UK \\ ${ }^{3}$ Department of Medical Physics and Clinical Engineering, Royal Hallamshire Hospital, Sheffield, UK
}

\section{Edited by:}

Laurel J. Trainor, McMaster University,

Canada

\section{Reviewed by:}

Jessica A. Grahn, MRC Cognition and

Brain Sciences Unit, UK

Ramesh Balasubramaniam,

McMaster University, Canada

${ }^{*}$ Correspondence:

Manon Grube, Newcastle Auditory

Group, Institute of Neuroscience,

Newcastle University Medical School,

Framlington Place, Newcastle upon

Tyne NE2 4HH, UK.

e-mail:manon.grube@ncl.ac.uk
Cerebellar functions in two types of perceptual timing were assessed: the absolute (durationbased) timing of single intervals and the relative (beat-based) timing of rhythmic sequences. Continuous transcranial magnetic theta-burst stimulation (cTBS) was applied over the medial cerebellum and performance was measured adaptively before and after stimulation. A large and significant effect was found in the TBS $(n=12)$ compared to the SHAM $(n=12)$ group for single-interval timing but not for the detection of a regular beat or a deviation from it. The data support the existence of distinct perceptual timing mechanisms and an obligatory role of the cerebellum in absolute interval timing with a functional dissociation from relative timing of interval within rhythmic sequences based on a regular beat.

Keywords: cerebellum, transcranial magnetic theta-burst stimulation, perception, timing, absolute, relative, duration, beat

\section{INTRODUCTION}

The current understanding of cerebellar functions includes motor learning (Glickstein, 1992; Thach et al., 1992) motor timing (Ivry et al., 1988; Ivry and Keele, 1989; Hore et al., 1991), sensory timing in motor control (Gao et al., 1996; Ivry et al., 2002; Koekkoek et al., 2003) and sensory timing in purely perceptual tasks (Gao et al., 1996), the focus of the present work. Perceptual timing of sensory input is particularly important in the auditory modality where the processing of speech and music requires the absolute, duration-based timing of intervals relevant to phoneme identification and the relative timing of intervals based on a regular beat as in musical rhythm (Klatt, 1976; Fraisse, 1984; Povel and Essens, 1985; Rosen, 1992; Patel et al., 2005). Recent studies in neuropsychology (Ivry and Keele, 1989; Nichelli et al., 1996; Malapani et al., 1998; Mangels et al., 1998; Harrington et al., 2004), functional imaging (Jueptner et al., 1995; Penhune et al., 1998; Mathiak et al., 2004; Xu et al., 2006; Grahn and Brett, 2007; Chen et al., 2008) and transcranial magnetic stimulation (Koch et al., 2007; Lee et al., 2007) implicate the cerebellum in perceptual and motor timing using tasks of absolute or relative timing (for recent review see Koch et al., 2009). The involvement of the cerebellum in the timing network of the brain is widely accepted, but the understanding of the mechanisms of timing still limited (Ivry and Schlerf, 2008). Models of perceptual timing suggest the existence of duration-based (or interval) and beatbased (entrainment) mechanisms (Pashler, 2001; McAuley and Jones, 2003; Jones and McAuley, 2005), supported by behavioral data from previous psychophysical studies (Monahan and Hirsh, 1990; Rammsayer and Brandler, 2004; Foxton et al., 2006; Grube and Griffiths, 2009). The neural substrates of this dissociation remain to be identified.
We propose here a functional dissociation of absolute, durationbased and relative, beat-based timing, and hypothesize that the cerebellum performs an obligatory role specific to absolute timing. The role of the cerebellum in the perceptual timing of single intervals and rhythmic sequences was tested by the assessment of the effect of continuous transcranial magnetic theta-burst stimulation (cTBS) (Huang et al., 2005). Stimulation was applied over the medial cerebellum, which has been reported to be a suitable site to interfere with perceptual timing in previous studies using other tasks and traditional rTMS (Koch et al., 2007; Lee et al., 2007). The effect was tested for three auditory timing tasks, including one of absolute timing of single intervals and two of relative timing that allowed time-interval measurement based on a regular beat. The study allows the direct assessment of (i) the specific cerebellar "stopwatch" role in absolute, duration-based timing of individual auditory events and (ii) the functional dissociation from the relative, beat-based timing of rhythmic sequences. The hypothesized effect of TBS as a test of cerebellar function was an acute interference with duration - but not beat-based timing that would parallel the same dissociation found in chronic lesion due to cerebellar degeneration (Grube et al., 2010).

\section{MATERIALS AND METHODS SUBJECTS}

The TBS group included 12 males of $23.5 \pm 4.5$ years of age; the matched SHAM group included 12 males of $22.3 \pm 4.2$ years of age. All participants were students from the University of Sheffield and right-handed as assessed by the Edinburgh Handedness Inventory (Oldfield, 1971). The study was approved of by the ethics committee of the University of Sheffield and all participants gave written informed consent. 


\section{TBS PROCEDURE}

The TBS setup consisted of a Super Rapid magnetic stimulator (Magstim UK) with a $70 \mathrm{~mm}$ figure-of-eight coil. The positioning of the coil was adopted from a previous study demonstrating efficient intervention with cerebellar processing (Lee et al., 2007). Stimulation was applied over the medial cerebellum, $1 \mathrm{~cm}$ below the inion used as the external anatomical landmark for guidance as in Lee et al. (2007). The region beneath the center of the coil corresponded to the vermal part of the inferior edge of the lobule VI bordering lobule VIIA (Schmahmann et al., 2000). Stimulation was performed at an intensity of $80 \%$ of the resting motor threshold (Rossini et al., 1994; Lee et al., 2007). Resting motor threshold was individually determined as the level which reliably induces a finger movement in 50\% of the stimulations according to Pridmore et al. (1998). Based on the physical distance between the scalp and the brain surface, the estimated effect of cerebellar stimulation would amount to about $75 \%$ of that of the identical stimulation applied to the motor cortex (Del Olmo et al., 2007). The coil was positioned tangentially to the scalp with the coil handle pointing upwards for effective TBS stimulation, and at $90^{\circ}$ angle for SHAM stimulation. Magnetic stimulation was applied following a powerful protocol of continuous TBS, which has previously been shown to be more efficient than traditional rTMS (Huang et al., 2005). TBS consists of bursts of three pulses delivered at 20 -ms intervals $(50 \mathrm{~Hz})$, that are repeated every $200 \mathrm{~ms}(5 \mathrm{~Hz})$ and was applied continuously for a 40-speriod, equaling 600 pulses in total. The estimated duration of the TBS effect was based on our previous experience with cerebellar stimulation (Lee et al., 2007; Bijsterbosch et al., 2010) and the effects of the same protocol of $40 \mathrm{~s}$ cTBS applied to the motor cortex lasting for $1 \mathrm{~h}$ (Huang et al., 2005); total testing time for all four tasks was confined to less than $30 \mathrm{~min}$. During and following TBS, none of the subjects reported any adverse effects. Some subjects experienced mild neck muscle contraction during TBS. Contraction of superficial musculature is experienced wherever TMS is applied to the cerebrum and does not interfere with the effect on the target area. We chose the inion as a site that has minimum muscle depth whilst at the same time providing access to the underlying cerebellum.

\section{PSYCHOACOUSTIC TESTING}

Tests were based on a two-alternative forced-choice paradigm following a two-down-one-up adaptive threshold tracking algorithm (Levitt, 1971). Each test included a minimum of three practice trials to familiarize the subject with the task, followed by 50 test trials; each trial contained one reference and one target stimulus. The position of the target was pseudo-randomized at equal probabilities across trials and fixed across subjects. Subjects communicated their response by pressing corresponding buttons on a response box. The difference between target and reference was well-above average thresholds initially and was adaptively decreased and increased after every two consecutive correct responses and one incorrect response, respectively. A larger initial step size was used up to the fourth reversal, i.e., a change from decrease to increase or vice versa; after that a smaller step size was used. Interstimulusand intertrial-intervals were $1500 \mathrm{~ms}$ each. Response time was not limited, but subjects were encouraged to make immediate decisions. Discrimination thresholds were calculated as the mean of the last six reversals, i.e., as the $70.9 \%$ correct point of the psychometrical function (Levitt, 1971). Each subject performed the four tasks before and immediately after magnetic stimulation; the order of tasks was systematically rotated between subjects. Total session duration was less than $2 \mathrm{~h}$, including explanation, pre- and poststimulation testing (less than $30 \mathrm{~min}$ each), magnetic stimulation and short breaks.

Stimuli were created and delivered using Matlab 6.5 (The Mathworks) with $44.1 \mathrm{kHz}$ sampling rate and 16-bit resolution. The stimuli were delivered at $70 \mathrm{~dB}$ rms Sound Pressure Level via an external soundcard (Edirol Audio Capture UA-3FX) and closed headphones (Sennheiser HD265 linear). All stimuli were composed of $200 \mathrm{~Hz}$ pure tones of $100 \mathrm{~ms}$ in duration including $20 \mathrm{~ms}$ gating times.

\section{Single-interval duration discrimination}

Subjects were required to discriminate between a longer target and a shorter reference interval of variable duration (Figure 1A). Intervals were marked by pairs of tones; references had inter-onset-intervals of $300,360,420,480,560$, or $600 \mathrm{~ms}$, presented at equal probabilities in a fixed randomized order. The initial difference in duration was $90 \%$ of the reference interval; the large and the small step size were $12 \%$ and $6 \%$, respectively.

\section{Regularity detection (Reg)}

Subjects were required to discriminate between a more regular target sequence and an irregular reference sequence based on the detection of the underlying regular beat of $400 \mathrm{~ms}$ (Figure 1B). Sequences were composed of 11 tones. The reference had a mean irregularity of $30 \%$, rendering the underlying pulse imperceptible (Madison and Merker, 2002). The target had an initial mean irregularity of $0 \%$, which was adaptively controlled in steps of $4 \%$ and $2.5 \%$. Irregularity was introduced by randomized shortenings and lengthenings of individual intervals, evenly distributed in the range of the mean $\pm 50 \%$ of the mean.

\section{Isochrony-deviation detection (Iso)}

Subjects were required to detect a lengthening of one time interval within a sequence based on an otherwise isochronous beat (Figure 1C). The reference sequence consisted of five tones at inter-onset-intervals of $300 \mathrm{~ms}$, the target included a lengthening of the third interval. Initial difference and step sizes were 60,6 , and $2 \mathrm{~ms}$, respectively.

\section{Intensity discrimination}

Subjects were required to discriminate between a louder target and a softer reference tone. The initial difference was $7 \mathrm{~dB}$, step sizes were 0.75 and $0.25 \mathrm{~dB}$.

\section{STATISTICAL ANALYSIS}

Distribution of data samples was tested by the Lilliefors-corrected Kolmogorov-Smirnov test for normal distribution. All data were almost exclusively normally distributed and variance was equal between samples with only minor deviations, and parametric tests were used to analyze effects of TBS vs. SHAM stimulation. Effects were investigated by calculating the individual ratios of post- vs. pre-stimulation thresholds, where a ratio greater than 
unity indicates a drop in performance and a ratio smaller than unity an improvement in performance. Between-group comparisons of effects of TBS vs. SHAM stimulation were carried out by the independent $t$-test, within-group effects by the paired $t$-test; significance level was $p=0.05$.

\section{RESULTS}

The effect of cerebellar TBS in comparison to SHAM stimulation was measured for the timing of single intervals (absolute timing task), the detection of a regular beat (relative timing task), and the detection of a deviation from an isochronous beat (relative timing task) (Figure 1). A non-timing control task of intensity discrimination was administered alongside. Thresholds were compared before (pre) and after (post) stimulation, and the effect of stimulation was calculated as the ratio of post- vs. pre-stimulation thresholds for the application of TBS $(n=12)$ or SHAM $(n=12)$. A significant effect of TBS compared with SHAM stimulation was found for the absolute, duration-based but not for the relative, beat-based timing tasks.

\section{SINGLE-INTERVAL DURATION DISCRIMINATION}

In the single-interval timing task, subjects had to discriminate between a longer target and a shorter reference interval of variable subsecond duration (Figure 1A, Var). In agreement with our a priori hypotheses, there was a consistent increase in thresholds in the TBS group but not in the SHAM group (significant at the level $p<0.05$, paired $t$-test; Figure 2A), and there was a significant difference in the post/pre threshold ratio in the TBS compared to the SHAM group (Figure 3A; significant at the level of $p<0.05$, independent $t$-test).

\section{REGULARITY DETECTION}

This task required the detection of a regular beat despite the introduction of an increasing amount of irregularity in an 11-tone sequence (Figure 1B, Reg). Based on the hypothesis that mechanisms for absolute and relative timing are distinct, the detection of a regular beat was not expected to depend critically on the absolute timing of single intervals and thus not expected to be affected by cerebellar TBS. There was no effect of stimulation on thresholds in the TBS or SHAM group (not significant at the level $p<0.05$, paired $t$-test; Figure $2 \mathbf{B}$ ) and no significant difference in the post vs. pre threshold-ratio between groups (Figure 3B; not significant at the level of $p<0.05$, independent $t$-test).

\section{ISOCHRONY-DEVIATION DETECTION}

In this task, subjects were required to detect an increase in the duration of one interval of an otherwise isochronous sequence (Figure 1C, Iso). The detection of a deviation from isochrony was hypothesized to be based on the timing of intervals relative to the beat rather than on the absolute timing of single intervals. Hence, as expected, there was no significant increase in thresholds in either group (not significant at the level $p<0.05$, paired $t$-test; Figure $2 \mathrm{C}$ ) and no difference found in the pre/post threshold-ratios between groups (Figure 3C; not significant at the level of $p<0.05$, independent $t$-test).

\section{INTENSITY DISCRIMINATION}

In this non-timing control task, where subjects had to identify a louder target against a softer reference tone, there was no systematic TBS or SHAM effect on thresholds (not significant at the level $p<0.05$, paired $t$-test) and no difference in the post/pre threshold-ratios between groups (not significant at the level of $p<0.05$, Mann-Whitney $U$ test).

\section{DISCUSSION}

The present work tested the role for the cerebellum in two types of perceptual timing by comparing task performance before and after cTBS over the medial cerebellum. A significant effect of TBS compared to SHAM stimulation was shown for the absolute, durationbased timing of single intervals but not for the relative, beat-based timing of intervals within rhythmic sequences.

\section{TESTING TIMING FUNCTIONS IN THE CEREBELLUM}

The present work used cTBS to test the cerebellar role in durationand beat-based timing. It built upon previous reports of: (i) the dissociation of duration - from beat-based timing in findings in patients with cerebellar degeneration (Grube et al., 2010), (ii) the same degeneration progressing from superior-anterior to

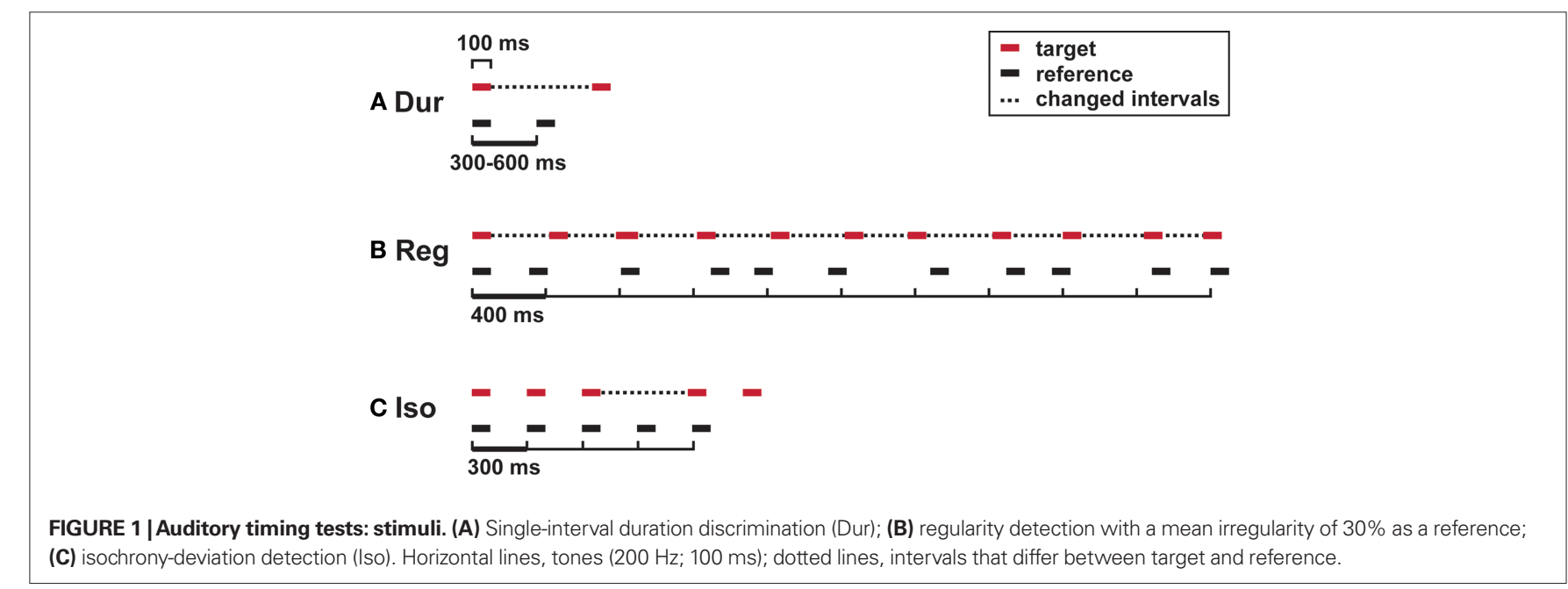




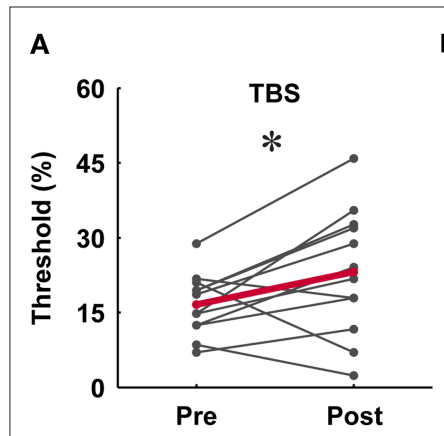

Dur

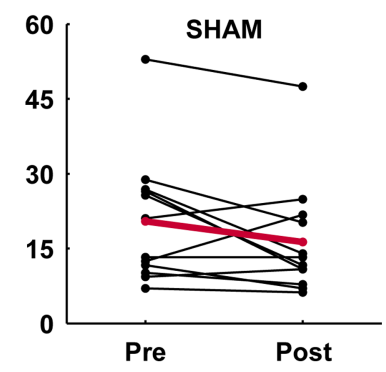

B

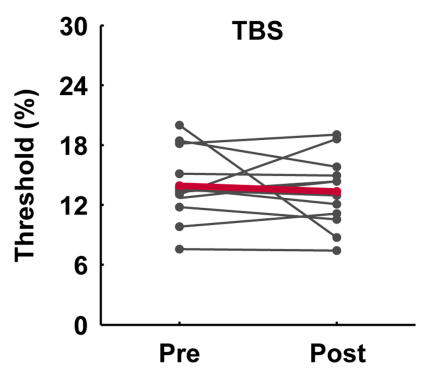

Reg

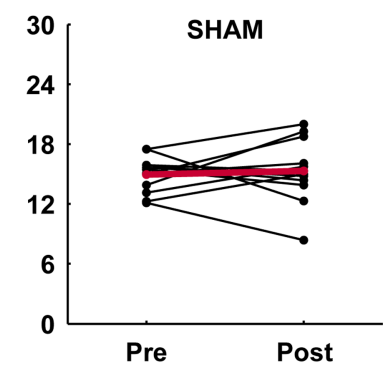

C

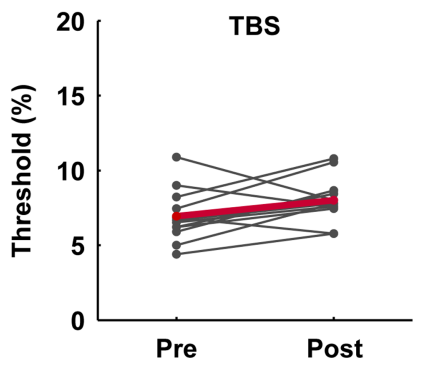

Iso

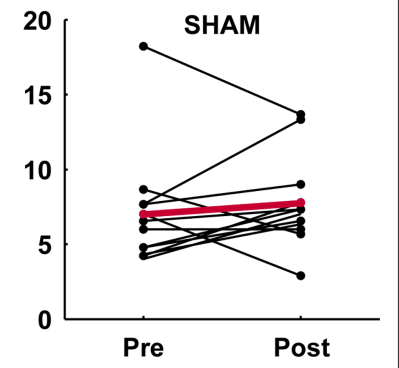

FIGURE 2 | Individual pre- and post-stimulation thresholds for the three timing tasks in the TBS and SHAM group. (A) Single-interval duration discrimination (Dur), (B) regularity detection (Reg), (C) isochrony-deviation detection (Iso). There was a significant effect of TBS on duration discrimination thresholds ( $p<0.05$; paired $t$-test) (A) but no significant effect on those for the other tasks; SHAM stimulation had no significant effect on any of the tasks.

inferior-posterior and most severely affecting the vermis (Butteriss et al., 2005), (iii) the effect of medial cerebellar rTMS on subsecond timer-interval perception (Lee et al., 2007), and (iv) the activation of the cerebellar vermis in functional imaging (Jueptner et al., 1995; Penhune et al., 1998).

The cerebellum is a deep structure (compared with cortical areas) that can be stimulated successfully (Miall, 2001; Bolognini and Ro, 2010), as has been demonstrated by a number of previous studies (Theoret et al., 2001; Del Olmo et al., 2007; Koch et al., 2007; Lee et al., 2007; Bijsterbosch et al., 2010). Stimulation here was applied over the medial cerebellum in the same location as in a previous study using rTMS (Lee et al., 2007). The spatial spread of the effect of present protocol of medial cerebellar cTBS would be expected to extend up to approximately $2 \mathrm{~cm}$ laterally toward the cerebellar hemispheres, with those areas underlying an effect of about $50 \%$ of

that at the center of stimulation, i.e., the vermal part of the inferior edge of the lobule VI bordering lobule VIIA; this estimate is based on ongoing modeling work in our group (unpublished data) and using a thresholding algorithm that is in accord with other established models (Ruohonen and Ilmoniemi, 1998).

We investigated the effect of medial cerebellar stimulation on distinct types of perceptual timing, and the data support a role for the cerebellum in duration-based timing, in particular the possible involvement of the vermis in accord with the impairment found after cerebellar degeneration (Grube et al., 2010). They are consistent with a central-to-right-lateralized function as suggested previously based on the effects of rTMS (Lee et al., 2007) and cerebellar stroke (Harrington et al., 2004); they do not allow a direct interpretation of a medial-lateral dissociation as suggested for motor timing functions (Ivry et al., 1988) or of the relevance of the activations of lateral hemispheres in imaging studies (Wiener et al., 2010).

Our principal finding implicates the cerebellum in inter-onset timing and specifically in absolute, duration-based timing. This is consistent with its suggested role in "event timing" and its suitability for the neural basis of a representation of time (Ivry, 1996; Ivry et al., 2002).

\section{A CEREBELLAR STOPWATCH}

The data support the hypothesis that the cerebellum has an obligatory role in the establishment of the representation of time intervals, in other words a stopwatch-like timing mechanism for measuring and storing the absolute duration of subsecond time intervals. The neural circuitry of the cerebellum is suitable for precise interval timing (Medina et al., 2000; Middleton and Strick, 2001; Middleton et al., 2008; Habas et al., 2009; Stoodley and Schmahmann, 2009; O'Reilly et al., 2010), and the anatomical connections with the basal ganglia and prefrontal cortex (via the thalamus) would allow the integration within the timing network of the brain (Middleton and Strick, 1994, 2001; Voogd and Glickstein, 1998; Hoshi et al., 2005). Recent functional data suggest specialized loops or zones of cortico-cerebellar connectivity, including one for sensori-motor functions involving cerebellar lobules V-VII (Habas et al., 2009; O'Reilly et al., 2010). The fact that stimulation in our study was centered on lobules VI and VIIA in the vermis would be consistent with the suggested timing mechanism being part of those sensori-motor functions. The present findings support the notion of a cerebellar neural substrate for the perception of subsecond time-intervals (Lee et al., 2007), and furthermore argue for a specialization on those time-intervals that are not part of a regular sequence. The consistent effect of medial cerebellar stimulation in the present and the previous study by Lee et al. (2007) suggests that the cerebellum plays an obligatory role in subsecond time-interval perception for empty intervals (the present study), as well as for filled intervals (Lee et al., 2007). This would support a common neural substrate for the timing of the absolute duration of auditory events (e.g., as in tone duration) and the timing between auditory events (e.g., as in the inter-onset-interval duration within a pair of tones).

\section{ABSOLUTE TIMING}

The interference of TBS with the absolute timing of single intervals of variable subsecond duration strongly supports an obligatory role for the cerebellum in the establishment of an internal perceptual 


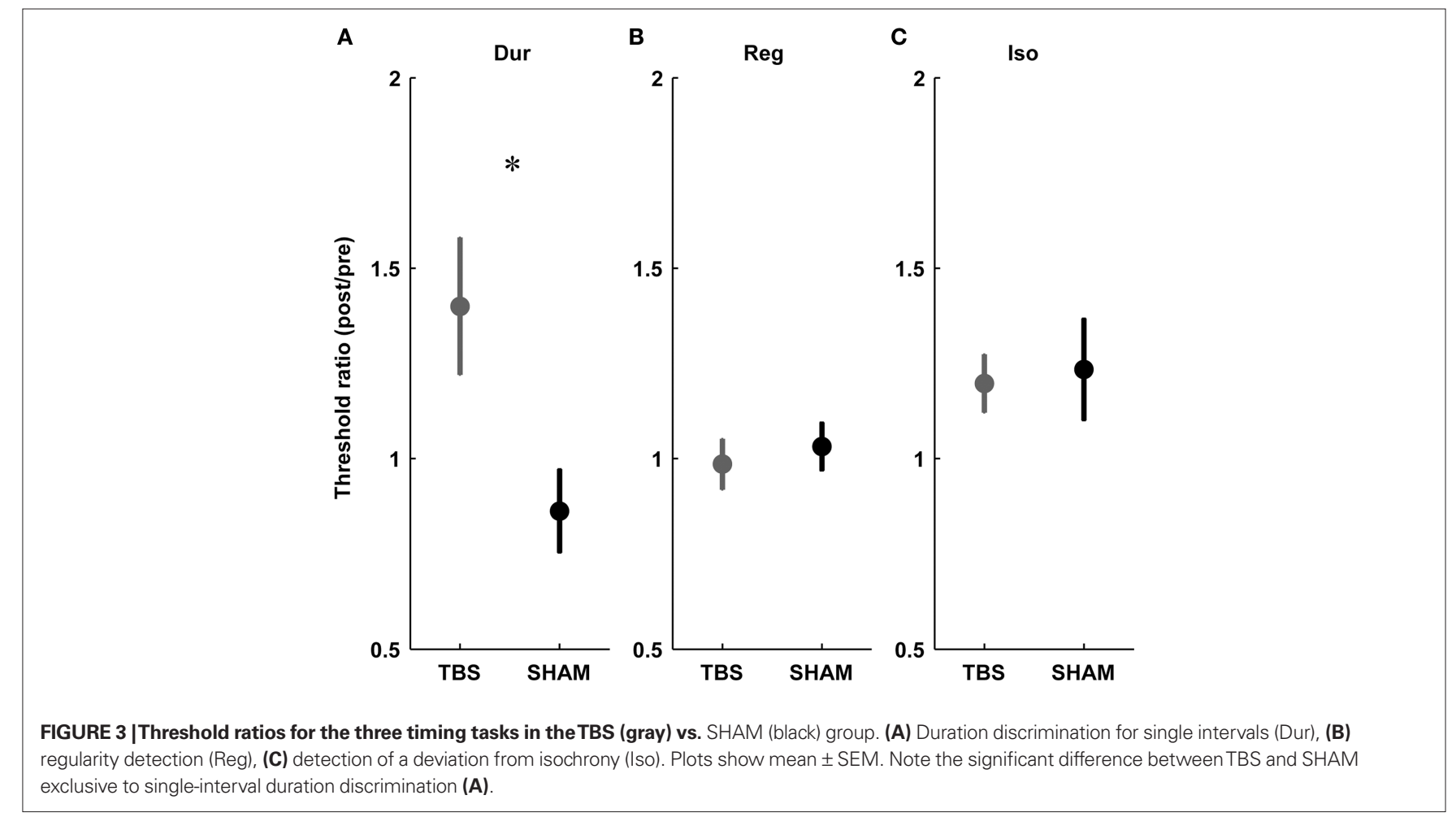

representation of the absolute duration of time intervals. The significant deficit in the absolute timing of single subsecond time intervals is consistent with findings from previous cerebellar TMS studies using other perceptual timing tasks such as interval bisection for perceived tone duration (Lee et al., 2007) and a reproduction task based on visual duration perception (Koch et al., 2007). The interference with the perceptual interval-timing between auditory events demonstrated here compares very well with neuropsychological reports of patients with cerebellar damage demonstrating an impairment in similar tasks (Ivry and Keele, 1989; Nichelli et al., 1996; Malapani et al., 1998; Mangels et al., 1998; Harrington et al., 2004). The present effect size is considerable and could be due to (i) the TBS protocol having induced a greater and longer-lasting neuronal response than traditional rTMS (Huang et al., 2005), (ii) the method of adaptive tracking of thresholds providing a reliable and robust measure at the level of the individual, and (iii) the use of a variable rather than a fixed reference interval requiring the immediate timing of two novel intervals per trial (compared with a repeated presentation throughout the task allowing the formation of a long-term representation).

\section{RELATIVE TIMING BASED ON A REGULAR BEAT}

The cerebellum has previously been implicated in beat-based timing based on functional activation during listening to rhythmic sequences (Penhune et al., 1998; Xu et al., 2006; Grahn and Brett, 2007; Chen et al., 2008) and impairments in motor entrainment after cerebellar rTMS (Theoret et al., 2001; Del Olmo et al., 2007) and chronic lesions (Ivry and Keele, 1989; Spencer et al., 2003; Harrington et al., 2004). However, neither type of evidence allows a clear inference about the role of the cerebellum in beat-based perceptual timing: motor entrainment to auditory sequences may be impaired independently of perceptual timing, and functional brain imaging data do not control the use of relative or absolute timing strategies and remain ambiguous with respect to the functional relevance of activations. The present study used TBS to acutely interfere with cerebellar processing during two beat-based perceptual timing tasks, i.e., the detection of regularity and the deviation from isochrony, which could both be achieved using relative timing: those tasks were not expected to depend on the accurate representation of absolute durations of individual intervals. In accord with the hypothesized dissociation between mechanisms of absolute and relative timing there was no discernable TBS effect shown for the two beat-based tasks in contrast to that shown for the absolute task. Theoretically, the suggested cerebellar "stopwatch" mechanism of absolute timing could be employed for the serial, duration-based encoding of the intervals of any sequence. In the beat-based sequences of the present study, those for regularity detection and isochrony violation detection were based on 10 and four intervals, respectively, and one might argue for the number of intervals as the reason for the difference in performance and effect of TBS. However, if either beat-based task was achieved by using the suggested cerebellar mechanism of duration-based timing, one would expect a significant effect of TBS on that task also. This was not the case, supporting the existence of an undisturbed alternative mechanism that allows beat-based timing to function independently of duration-based timing. This dissociation does not imply that these mechanisms are mutually exclusive. We would support the notion that both types of timing would operate at least initially in any of the tasks, and take up their functional relevance during the unfolding of the stimulus: the duration-based mechanism for the single intervals, and the beat-based one for the regular sequences. The present perceptual assessment of beat-based and 
single-interval timing demonstrates the functional dissociation between the two and the absence of an effect supports our a priori hypothesis that beat-based timing within rhythmic sequences does not depend critically on the absolute timing of single intervals by the cerebellum. The demonstrated dissociation suggests a distinct neural substrate for beat-based perceptual timing that may not be located in the cerebellum. The demonstrated preservation of performance despite acute application of cerebellar TBS is consistent that of sensori-motor transduction of beat-based entrainment in a previous neuropsychological study of timing in patients with chronic cerebellar damage (Molinari et al., 2005).

\section{PERCEPTUAL VS. MOTOR ENTRAINMENT}

The present findings do not contradict previous neuropsychological and functional evidence of cerebellar involvement in regular motor timing functions (Ivry and Keele, 1989; Ivry and Richardson, 2002; Spencer et al., 2003; Harrington et al., 2004; Del Olmo et al., 2007; Thaut et al., 2009). The previous neuropsychological reports argue mainly for deficits in the implementation of individual intervals which could be interpreted as a deficit in the discrete timing of individual events (Ivry et al., 2002) started anew by every beat from a central timekeeper that entrains with a regular beat (Wing and Kristofferson, 1973). Suggestions of cerebellar involvement in the central timekeeper (Ivry et al., 1988; Molinari et al., 2005) could be reconciled with the present model if perceptual and motor entrainment were not subserved by the same mechanism. We cannot exclude the possibility that certain sites, e.g., in lateral parts of the cerebellar hemispheres, that were unaffected in the present study may be involved in beat-based perceptual timing. Given the lack of evidence from previous studies, such a functional dissociation within the cerebellum remains speculative. Recent neuropsychological (Grahn and Brett, 2009) and functional imaging studies (Grahn and Brett, 2007; Chen et al., 2008; Thaut et al., 2009) support the alternative possibility that the neural substrate for beat-based perceptual timing may found elsewhere and implicate the basal ganglia and prefrontal cortex.

\section{STIMULUS DESIGN: A POTENTIAL CONFOUND}

One possible confound that needs to be considered as an explanation for the dissociation of the TBS-induced effect on absolute but not relative timing relates to the total duration of the stimuli and the possible distinction of subsecond vs. suprasecond timing. A specific

\section{REFERENCES}

Bijsterbosch, J. D., Lee, K. H., Hunter, M. D., Tsoi, D. T., Lankappa, S., Wilkinson, I. D., Barker, A. T., and Woodruff, P. W. (2010). The role of the cerebellum in sub- and supraliminal error correction during sensorimotor synchronization: evidence from fMRI and TMS. J. Cogn. Neurosci. doi: 10.1162/ jocn.2010.21506 [Epub ahead of print].

Bolognini, N., and Ro, T. (2010). Transcranial magnetic stimulation:

involvement of the cerebellum has been suggested for the subsecond range (Lewis and Miall, 2003), with a possible gradual transition to the suprasecond range (Lewis and Miall, 2006). Previous TMS studies have shown that rTMS applied to the cerebellum did not affect perception of suprasecond time intervals (Lee et al., 2007; Koch et al., 2007), and neuropsychological reports on the effect of chronic cerebellar damage have further supported this (Mangels et al., 1998; Gooch et al., 2010). The total duration of stimuli in the present work varied from a few hundred milliseconds in the single-interval task, to just over $1 \mathrm{~s}$ and up to several seconds in the isochrony and regularity tasks, respectively. Hence, one might conclude that the lack of TBS effects on the regularity and isochrony tasks might be due to the longer stimulus duration in these tasks than in the single-interval task. However, the duration of intervals that needed to be timed was in the subsecond range for all three tasks, i.e., 300 to over $600 \mathrm{~ms}$ ( $\max .870 \mathrm{~ms}$ for the single-interval, $400 \mathrm{~ms}$ on average for the regularity and $300 \mathrm{~ms}$ or more in the isochrony one. The variation occurred in the duration of the context that these intervals were presented in but not in the duration of the intervals themselves. Hence, the relative, beat-based timing tasks were tests of subsecond and not of suprasecond time-interval perception and a dissociation in the use of mechanisms compared to that of absolute, duration-based subsecond time perception was shown.

\section{CONCLUSION}

The present work tested the cerebellum as part of the timing network of the human brain using cTBS. This is the first study to systematically assess the acute effect of cerebellar TBS on two postulated types of perceptual timing: duration-based and beat-based timing. The data demonstrate a specific cerebellar function subserving the absolute, duration-based perception of single time-intervals without a requirement for an obligatory cerebellar involvement in the relative, beat-based timing of rhythmic sequences. The findings support the existence of distinct mechanisms and neural substrates for the absolute, duration-based and the relative, beat-based perceptual timing of intervals.

\section{ACKNOWLEDGMENTS}

This work is supported by a Wellcome Trust Grant and an Ataxia UK Grant. The authors thank J. Youngs and F. Peerally for help with testing and S. Teki for commenting on previous versions of the manuscript.

mic finger movements. J. Neurophysiol. 98, 145-152.

Foxton, J. M., Nandy, R. K., and Griffiths, T. D. (2006). Rhythm deficits in 'tone deafness'. Brain Cogn. 62, 24-29.

Fraisse, P. (1984). Perception and estimation of time. Annu. Rev. Psychol. 35, $1-36$.

Gao, J. H., Parsons, L. M., Bower, J. M., Xiong, J., Li, J., and Fox, P. T. (1996). Cerebellum implicated in sensory acquisition and discrimination rather than motor control. Science 272, 545-547.
Glickstein, M. (1992). The cerebellum and motor learning. Curr. Opin. Neurobiol. 2, 802-806.

Gooch, C. M., Wiener, M., Wencil, E. B., and Coslett, H. B. (2010). Interval timing disruptions in subjects with cerebellar lesions. Neuropsychologia 48, 1022-1031.

Grahn, J. A., and Brett, M. (2007). Rhythm and beat perception in motor areas of the brain. J. Cogn. Neurosci. 19, 893-906.

Grahn, J. A., and Brett, M. (2009). Impairment of beat-based rhythm 
discrimination in Parkinson's disease. Cortex 45, 54-61.

Grube, M., Cooper, F. E., Chinnery, P. F., and Griffiths, T.D. (2010). Dissociation of duration-based and beat-based auditory timing in cerebellar degeneration. Proc. Natl. Acad. Sci. U.S.A. 107, 11597-11601.

Grube, M., and Griffiths, T. D. (2009). Metricality-enhanced temporal encoding and the subjective perception of rhythmic sequences. Cortex $45,72-79$.

Habas, C., Kamdar, N., Nguyen, D., Prater, K., Beckmann, C. F., Menon, V., and Greicius, M. D. (2009). Distinct cerebellar contributions to intrinsic connectivity networks. J. Neurosci. 29, 8586-8594.

Harrington, D. L., Lee, R. R., Boyd, L. A., Rapcsak, S. Z., and Knight, R. T. (2004). Does the representation of time depend on the cerebellum? Effect of cerebellar stroke. Brain 127 (Pt 3), 561-574.

Hore, J., Wild, B., and Diener, H. C. (1991). Cerebellar dysmetria at the elbow, wrist, and fingers. J. Neurophysiol. 65, 563-571.

Hoshi, E., Tremblay, L., Feger, J., Carras, P. L., and Strick, P. L. (2005). The cerebellum communicates with the basal ganglia. Nat. Neurosci. 8, 1491-1493.

Huang, Y. Z., Edwards, M. J., Rounis, E., Bhatia, K. P., and Rothwell, J. C. (2005). Theta burst stimulation of the human motor cortex. Neuron 45, 201-206.

Ivry, R. B. (1996). The representation of temporal information in perception and motor control. Curr. Opin. Neurobiol. 6, 851-857.

Ivry, R. B., and Keele, S. W. (1989). Timing functions of the cerebellum. J. Cogn. Neurosci. 1, 136-152.

Ivry, R. B., Keele, S. W., and Diener, H. C. (1988). Dissociation of the lateral and medial cerebellum in movement timing and movement execution. Exp. Brain Res. 73, 167-180.

Ivry, R. B., and Richardson, T. C. (2002). Temporal control and coordination: the multiple timer model. Brain Cogn. 48, 117-132.

Ivry, R. B., and Schlerf, J. E. (2008). Dedicated and intrinsic models of time perception. Trends Cogn. Sci. 12, 273-280.

Ivry, R. B., Spencer, R. M., Zelaznik, H. N., and Diedrichsen, J. (2002). The cerebellum and event timing. Ann. N.Y. Acad. Sci. 978, 302-317.

Jones, M. R., and McAuley, J. D. (2005). Time judgments in global temporal contexts. Percept. Psychophys. 67, 398-417.

Jueptner, M., Rijntjes, M., Weiller, C., Faiss, J. H., Timmann, D., Mueller, S. P., and Diener, H. C. (1995). Localization of a cerebellar timing process using PET. Neurology 45, 1540-1545.

Klatt, D. H. (1976). Linguistic uses of segmental duration in English: acoustic and perceptual evidence. J. Acoust. Soc. Am. 59, 1208-1221.

Koch, G., Oliveri, M., and Caltagirone, C. (2009). Neural networks engaged in milliseconds and seconds time processing: evidence from transcranial magnetic stimulation and patients with cortical or subcortical dysfunction. Philos. Trans. R. Soc. Lond. B. Biol. Sci. 364, 1907-1918.

Koch, G., Oliveri, M., Torriero, S., Salerno, S., Gerfo, E. L., and Caltagirone, C. (2007). Repetitive TMS of cerebellum interferes with millisecond time processing. Exp. Brain Res. 179, 291-299.

Koekkoek, S. K., Hulscher, H. C., Dortland, B. R., Hensbroek, R. A., Elgersma, Y., Ruigrok, T. J., and De Zeeuw, C. I. (2003). Cerebellar LTD and learning-dependent timing of conditioned eyelid responses. Science 301, 1736-1739.

Lee, K. H., Egleston, P. N., Brown, W. H., Gregory, A. N., Barker, A. T., and Woodruff, P. W. (2007). The role of the cerebellum in subsecond time perception: evidence from repetitive transcranial magnetic stimulation. J. Cogn. Neurosci. 19, 147-157.

Levitt, H. (1971). Transformed up-down methods in psychoacoustics. J. Acoust. Soc. Am. 49, 467-477.

Lewis, P. A., and Miall, R. C. (2003). Distinct systems for automatic and cognitively controlled time measurement: evidence from neuroimaging. Curr. Opin. Neurobiol. 13, 250-255.

Lewis, P. A., and Miall, R. C. (2006). Remembering the time: a continuous clock. Trends Cogn. Sci. 10, 401-406.

Madison, G., and Merker, B. (2002). On the limits of anisochrony in pulse attribution. Psychol. Res. 66, 201-207.

Malapani, C., Dubois, B., Rancurel, G., and Gibbon, J. (1998). Cerebellar dysfunctions of temporal processing in the seconds range in humans. Neuroreport 9, 3907-3912.

Mangels, J. A., Ivry, R. B., and Shimizu, N. (1998). Dissociable contributions of the prefrontal and neocerebellar cortex to time perception. Brain Res. Cogn. Brain Res. 7, 15-39.

Mathiak, K., Hertrich, I., Grodd, W., and Ackermann, H. (2004). Discrimination of temporal information at the cerebellum: functional magnetic resonance imaging of nonverbal auditory memory. Neuroimage 21, 154-162.

McAuley, J. D., and Jones, M. R. (2003). Modeling effects of rhythmic context on perceived duration: a com- parison of interval and entrainment approaches to short-interval timing. J. Exp. Psychol. Hum. Percept. Perform. 29, 1102-1125.

Medina, J. F., Garcia, K. S., Nores, W. L., Taylor, N. M., and Mauk, M. D. (2000). Timing mechanisms in the cerebellum: testing predictions of a large-scale computer simulation. $J$. Neurosci. 20, 5516-5525.

Miall, C. (2001). Stimulating times for TMS. Trends Cogn. Sci. 5, 329.

Middleton, F. A., and Strick, P. L. (1994). Anatomical evidence for cerebellar and basal ganglia involvement in higher cognitive function. Science 266 458-461.

Middleton, F. A., and Strick, P. L. (2001). Cerebellar projections to the prefrontal cortex of the primate. J. Neurosci. 21, 700-712.

Middleton, S. J., Racca, C., Cunningham, M. O., Traub, R. D., Monyer, H., Knopfel, T., Schofield, I. S., Jenkins, A., and Whittington, M. A. (2008) High-frequency network oscillations in cerebellar cortex. Neuron 58 763-474.

Molinari, M., Leggio, M. G., Filippini, V., Gioia, M. C., Cerasa, A., and Thaut, M.H. (2005). Sensorimotor transduction of time information is preserved in subjects with cerebellar damage. Brain Res. Bull 67, 448-458.

Monahan, C. B., and Hirsh, I. J. (1990). Studies in auditory timing: 2 . Rhythm patterns. Percept. Psychophys. 47, 227-242.

Nichelli, P., Alway, D., and Grafman, J. (1996). Perceptual timing in cerebellar degeneration. Neuropsychologia 34 863-871.

Oldfield, R.C. (1971). The assessment and analysis of handedness: the Edinburgh inventory. Neuropsychologia 9, 97-113.

O'Reilly, J. X., Beckmann, C. F., Tomassini, V., Ramnani, N., and Johansen-Berg, H. (2010). Distinct and overlapping functional zones in the cerebellum defined by resting state functional connectivity. Cereb. Cortex 20 953-965.

Pashler, H. (2001). Perception and production of brief durations: beat-based versus interval-based timing. J. Exp. Psychol. Hum. Percept. Perform. 27, 485-493.

Patel, A. D., Iversen, J. R., Chen, Y., and Repp, B. H. (2005). The influence of metricality and modality on synchronization with a beat. Exp. Brain Res. 163, 226-238.

Penhune, V. B., Zattore, R. J., and Evans, A.C. (1998). Cerebellar contributions to motor timing: a PET study of auditory and visual rhythm reproduction. J. Cogn. Neurosci. 10, 752-765.
Povel, D. J., and Essens, P. (1985). Perception of temporal patterns. Music Percept. 2, 411-440.

Pridmore, S., Fernandes Filho, J. A., Nahas, Z., Liberatos, C., and George, M. S. (1998). Motor threshold in transcranial magnetic stimulation: a comparison of a neurophysiological method and a visualization of movement method. J. Ect. 14, 25-27.

Rammsayer, T.H., and Brandler, S. (2004). Aspects of temporal information processing: a dimensional analysis. Psychol. Res. 69, 115-123.

Rosen, S. (1992). Temporal information in speech: acoustic, auditory and linguistic aspects. Philos. Trans. R. Soc. Lond. B. Biol. Sci. 336, 367-373.

Rossini, P. M., Barker, A. T., Berardelli, A., Caramia, M. D., Caruso, G., Cracco, R. Q., Dimitrijevic, M. R., Hallett, M., Katayama, Y., Liicking, C. H., Maertens de Noordhout, A. L., Marsden, C. D., Murray, N. M. F., Rothwell, J. C., Swash, M., and Tomberg, C. (1994). Non-invasive electrical and magnetic stimulation of the brain, spinal cord and roots: basic principles and procedures for routine clinical application. Report of an IFCN committee. Electroencephalogr. Clin. Neurophysiol. 91, 79-92.

Ruohonen, J., and Ilmoniemi, R. J. (1998). Focusing and targeting of magnetic brain stimulation using multiple coils. Med. Biol. Eng. Comput. 36, 297-301.

Schmahmann, J.D., Doyon, J., Toga, A.W., Petrides, M., and Evans, A. C. (2000). MRI Atlas of the Human Cerebellum. San Diego: Academic Press.

Spencer, R. M., Zelaznik, H. N., Diedrichsen, J., and Ivry, R. B. (2003). Disrupted timing of discontinuous but not continuous movements by cerebellar lesions. Science 300 , 1437-1439.

Stoodley, C. J., and Schmahmann, J. D. (2009). Functional topography in the human cerebellum: a meta-analysis of neuroimaging studies. Neuroimage 44 489-501.

Thach, W. T., Goodkin, H. P., and Keating, J. G. (1992). The cerebellum and the adaptive coordination of movement. Annu. Rev. Neurosci. 15, 403-442.

Thaut, M.H., Stephan, K. M., Wunderlich, G., Schicks, W., Tellmann, L., Herzog, H., McIntosh, G. C., Seitz, R. J., and Homberg, V. (2009). Distinct cortico-cerebellar activations in rhythmic auditory motor synchronization. Cortex 45, 44-53.

Theoret, H., Haque, J., and Pascual-Leone, A. (2001). Increased variability of paced finger tapping accuracy following repetitive magnetic stimulation of the cerebellum in humans. Neurosci. Lett. 306, 29-32. 
Voogd, J., and Glickstein, M. (1998). The anatomy of the cerebellum. Trends Neurosci. 21, 370-375.

Wiener, M., Turkeltaub, P., and Coslett, H. B. (2010). The image of time: a voxelwise meta-analysis. Neuroimage 49, 1728-1740.

Wing, A., and Kristofferson, A. (1973). Response delays and the timing of discrete motor responses. Percept. Rychophys. 14, 5-12.
Xu, D., Liu, T., Ashe, J., and Bushara, K. O. (2006). Role of the olivo-cerebellar system in timing. J. Neurosci. 26, 5990-5995.

Conflict of Interest Statement: The authors declare that the research was conducted in the absence of any commercial or financial relationships that could be construed as a potential conflict of interest.
Received: 18 May2010; paperpending published: 21 May 2010; accepted: 25 September 2010; published online: 25 October 2010. Citation: Grube M, Lee K-H, Griffiths TD, Barker AT and Woodruff PW (2010) Transcranial magnetic theta-burst stimulation of the human cerebellum distinguishes absolute, duration-based from relative, beat-based perception of subsecond time intervals. Front. Psychology 1:171. doi: 10.3389/fpsyg.2010.00171
This article was submitted to Frontiers in Auditory Cognitive Neuroscience, a specialty of Frontiers in Psychology. Copyright (c) 2010 Grube, Lee, Griffiths, Barker and Woodruff. This is an openaccess article subject to an exclusive license agreement between the authors and the Frontiers Research Foundation, which permits unrestricted use, distribution, and reproduction in any medium, provided the original authors and source are credited. 